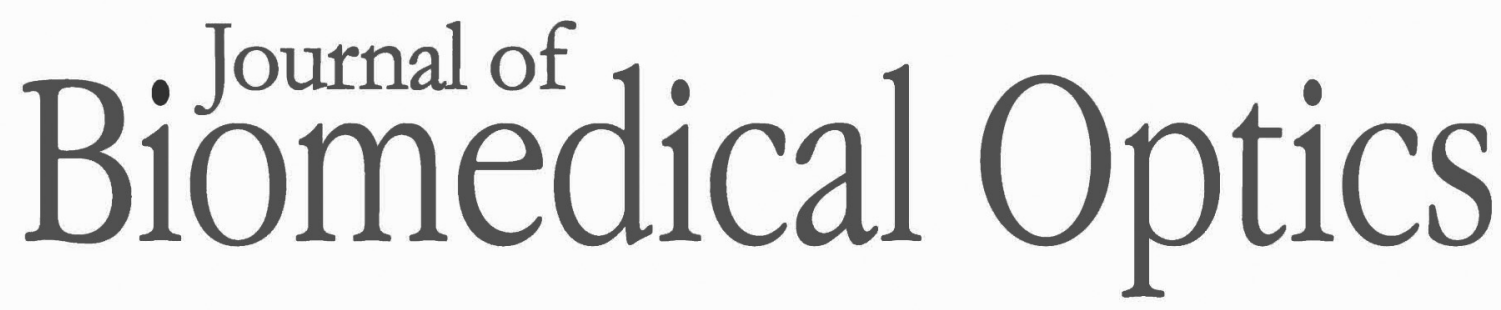

\title{
SPIEDigitalLibrary.org/jbo
}

\section{Label-free separation of human embryonic stem cells and their differentiating progenies by phasor fluorescence lifetime microscopy}

Chiara Stringari

Robert Sierra

Peter J. Donovan

Enrico Gratton 


\title{
Label-free separation of human embryonic stem cells and their differentiating progenies by phasor fluorescence lifetime microscopy
}

\author{
Chiara Stringari, ${ }^{a}$ Robert Sierra, ${ }^{\mathrm{b}}$ Peter J. Donovan, ${ }^{\mathrm{b}, \mathrm{c}}$ and Enrico Gratton ${ }^{\mathrm{a}}$ \\ a University of California, Irvine, Laboratory of Fluorescence Dynamics, Biomedical Engineering Department, Irvine, California \\ ${ }^{b}$ University of California, Irvine, Departments of Developmental \& Cell Biology and of Biological Chemistry, Irvine, California \\ 'University of California, Irvine, Sue and Bill Gross Stem Cell Research Center, Irvine, California
}

\begin{abstract}
We develop a label-free optical technique to image and discriminate undifferentiated human embryonic stem cells (hESCs) from their differentiating progenies in vitro. Using intrinsic cellular fluorophores, we perform fluorescence lifetime microscopy (FLIM) and phasor analysis to obtain hESC metabolic signatures. We identify two optical biomarkers to define the differentiation status of hESCs: Nicotinamide adenine dinucleotide $(\mathrm{NADH})$ and lipid droplet-associated granules (LDAGs). These granules have a unique lifetime signature and could be formed by the interaction of reactive oxygen species and unsaturated metabolic precursor that are known to be abundant in hESC. Changes in the relative concentrations of these two intrinsic biomarkers allow for the discrimination of undifferentiated hESCs from differentiating hESCs. During early hESC differentiation we show that NADH concentrations increase, while the concentration of LDAGs decrease. These results are in agreement with a decrease in oxidative phosphorylation rate. Single-cell phasor FLIM signatures reveal an increased heterogeneity in the metabolic states of differentiating $\mathrm{H} 9$ and $\mathrm{H} 1 \mathrm{hESC}$ colonies. This technique is a promising noninvasive tool to monitor hESC metabolism during differentiation, which can have applications in high throughput analysis, drug screening, functional metabolomics and induced pluripotent stem cell generation. (0) 2012 Society of Photo-Optical Instrumentation Engineers (SPIE). [DOI: 10.1117/1.JBO.17.4.046012]
\end{abstract}

Keywords: fluorescence lifetime microscopy; phasor analysis; stem cell; human embryonic stem cells; two-photon imaging; autofluorescence.

Paper 11598 received Oct. 12, 2011; revised manuscript received Feb. 23, 2012; accepted for publication Feb. 28, 2012; published online Apr. 20, 2012

\section{Introduction}

Human embryonic stem cells (hESCs) and induced pluripotent stem cells (hiPSCs) are unique in that they self-renew in culture while maintaining the ability to differentiate into all three germ layers mesoderm, ectoderm and endoderm - a property referred to as pluripotency. ${ }^{1-4}$ Thus, hESCs and hiPSCs offer a unique opportunity for drug discovery and toxicological screening, studying human development and developing treatments for human diseases. Since hESCs can be rapidly differentiated in vitro with addition of growth factors, they can provide an unlimited source of material for cell-replacement therapy to treat degenerative disease or traumatic injury.

However, the promising stem-cell research for tissue engineering and clinical application is limited by the difficulty to obtain pure cell lineages. hESC lines are known to be morphologically and phenotypically heterogeneous in culture; different hESC subpopulations have different functional properties such as growth, differentiation properties and different propensities for lineage selection upon differentiation. ${ }^{5,6}$ Stem cell differentiation in vitro generates heterogeneity, and differentiated populations are often characterized by a mixture of cells at different stages of maturation and with different patterns of gene expression. $^{7}$
Understanding the mechanisms and the extrinsic regulation of cell-fate decisions in stem cell populations and the identification and isolation of pure cell lineages are two major goals of stem biology. ${ }^{8}$ Therefore, there is a high demand for methods capable of determining the phenotype of cells and to identify and isolate cells of interest from a heterogeneous population. Methods such as immunohistochemistry, polymerase chain reaction (PCR), DNA/RNA microarrays and metabolic assays are currently used for identifying different cells' phenotypes and gene expression. However, these methodologies are time consuming and they impair the viability of the cells. These methods cannot be used for in vitro studies for clinical utility.

Classic hESC markers include alkaline phosphatase, the transcription factors OCT4, SOX2 and NANOG and a variety of glycolipid and transmembrane glycoprotein cell-surface markers ([SSEA]-3, [SSEA]-4, TRA-1-81, TRA-1-60). ${ }^{1,9-11}$

Cell-sorting techniques, which utilize monoclonal antibody for specific surface markers, are routinely used to identify pluripotent hESCs in vitro. Flow cytometry and fluorescence-activated cell sorting (FACS) provide separation of cellular populations based on fluorescent labeling of cell surface markers. ${ }^{12}$ Magnetic-activated cell sorting (MACS) instead allows separating cells by incubating magnetic nanoparticles coated with antibodies against a particular surface antigen. Although cell-sorting efficiencies are continuously being optimized, ${ }^{13-17}$ cell viability after sorting is still not very high, and this process has the capability of

0091-3286/2012/\$25.00 @ 2012 SPIE
Address all correspondence to: Enrico Gratton, University of California, Laboratory of Fluorescence Dynamics, Biomedical Engineering Department, Irvine, California. Tel.: 949-824-2674; Fax: 949-824-1727; E-mail: egratton22@yahoo.com. 
altering further differentiation of hESCs. Therefore, there is a high need for label-free and noninvasive techniques in this field. Some label-free techniques have been developed to sort stem cells from their differentiated progenies based on dielectric properties ${ }^{18}$ or chemical analysis by Raman spectroscopy. ${ }^{19-21}$

Multiphoton fluorescence microscopy (MPM) can also be used to image intrinsic fluorophores in live cells and tissues with minimal photo damage and phototoxicity. ${ }^{22-25}$ Fluorescence lifetime microscopy (FLIM) measures the time-decay characteristics of the cell and tissue microenvironment and allows molecular localization and identification of intrinsic fluorophores and endogenous proteins such as collagen, elastin, porphyrin, retinoids, flavins, nicotinamide adenine dinucleotide (NADH), hemoglobin and serotonin. Some intrinsic fluorophores have a physiological relevance and can be used as biomarkers; for example reduced NADH is an essential cofactor for oxidation-reduction (redox) reactions and oxidative phosphorylation and glycolysis. Biochemical estimation of NADH concentration has been extensively used to monitor cellular energy metabolism in vivo and in vitro, and FLIM has become a valuable technique to image life cells and to analyze metabolic states of cells. ${ }^{26-28}$ FLIM has been recently employed to image and to discriminate undifferentiated adult stem cells from differentiated cells in vitro. ${ }^{29-31}$

We recently showed that the phasor analysis of FLIM, is a very sensitive label-free and "fit-free" method to identify and distinguish different metabolic and differentiation states of germ cells in a living tissue. ${ }^{32}$

$\mathrm{hESC}$ have a unique metabolic signature associated with their phenotype. Metabolic patterns and changes are known to play an important role in stem-cell differentiation and pluripotency. ${ }^{33,34}$ The role of metabolic oxidation, oxidative stress and redox state has emerged as an important modulator of stem-cell fate decisions such as self-renewal/pluripotency, differentiation/lineage specification, programmed cell death and quiescence. ${ }^{34-40}$ Oxygen levels regulate mitochondrial metabolism, intracellular generation of reactive oxygen species (ROS) and ultimately the differentiation of stem cells. ${ }^{41,42}$ ROS are produced by the mitochondria during oxidative metabolism and ROS levels influence metabolite concentration, transcription-factor activity and other upstream signaling events involved in cell division, differentiation, survival and oncogenic transformation. ${ }^{36,43-45}$

hESCs appear to have higher mitochondrial activity than adult stem cells and differentiated cells, likely to be involved in supplying ATP for biomass production. ${ }^{46,47} \mathrm{hESCs}$ are characterized by a higher rate of oxidative phosphorylation with respect to glycolysis compared to adult stem cells ${ }^{46}$ and by the presence of unsaturated metabolites that are being oxidized during differentiation. ${ }^{34}$

In this work we image hESCs with FLIM, and using phasor analysis we directly map intrinsic fluorescent metabolic biomarkers of cells without prior assumption. ${ }^{32,48}$ Our results show that it is possible to monitor the metabolic activity of hESCs in a noninvasive way. We identify two intrinsic metabolic biomarkers whose levels change during hESC differentiation and provide an intrinsic contrast for the FLIM signature of differentiating hESCs. Label-free phasor analysis of FLIM measurements can distinguish undifferentiated stem cells from the differentiated progenies by their metabolic signatures. By cell phasor analysis of single hESCs, we measure high metabolic heterogeneity in $\mathrm{H} 9$ and $\mathrm{H} 1 \mathrm{hESC}$-lines during differentiation and throughout colonies in culture.

\section{Results}

\subsection{Identification of the Intrinsic Biomarkers in Human Embryonic Stem-Cell Colonies}

We separate and identify different intrinsic fluorescent metabolites in the hESC colonies by detecting clusters with different FLIM signatures within the phasor plot. After label-free FLIM imaging we perform retrospective in vitro staining or immunostaining after fixation to colocalize the FLIM signature of intrinsic fluorescent biomarkers with specific hESC compartments.

Figure 1 shows a representative image of the autofluorescence from a colony of undifferentiated H9 hESCs plated on a mouse-embryonic-fibroblast (MEF) feeder substrate. We perform the phasor transformation of the FLIM image of the hESC colony (see Sec. 4 and Ref. 32). Every pixel of the FLIM image is transformed into a pixel in the phasor plot. Figure 1(a) displays the phasor histogram distribution of the FLIM image of an undifferentiated hESC colony that is located inside the universal circle of the phasor plot, indicating the multiexponential characteristic of its decay. ${ }^{32}$ Within the phasor distribution we can identify three main clusters that correspond to different cell types and cell compartments [Fig. 1(a) and 1(d)]. hESC nucleus and
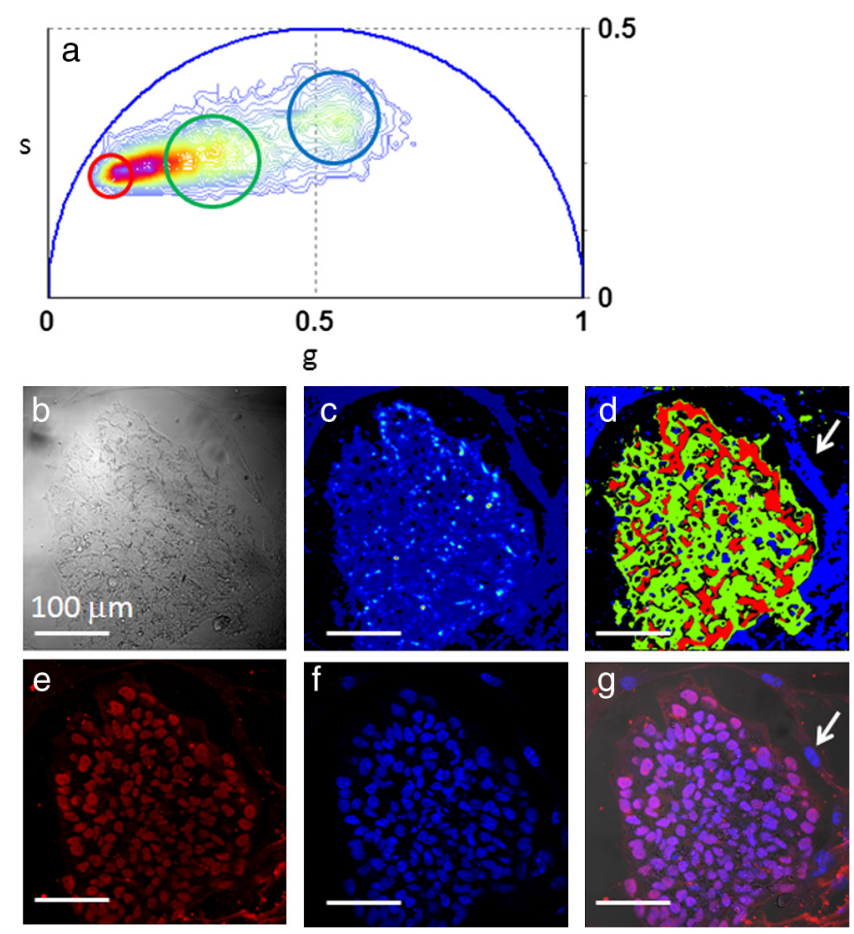

Fig. 1 Label-free identification of hESCs: (a) FLIM phasor histogram of the FLIM image excited at $760 \mathrm{~nm}$ from one H9 hESC colony cocultured with mouse embryonic fibroblasts (MEFs). The color scale (from blue to purple) corresponds to the 64 levels of the contours that indicate the percent occurrence in the phasor histogram of the pixels of the image. Different clusters within the phasor distribution correspond to bright lipid droplet-associated granules within hESCs (red), the hESCs themselves (green) and the MEF feeders (blue). Transmission image (a) and two-photon fluorescence intensity image (c) of a undifferentiated hESC colony grown on MEF feeders. (d) Phasor color map. Pixels of different colors correspond to the color of the cluster in the phasor plot A. Arrow indicates a MEF. (e) Expression of the pluripotency marker OCT4 in the same colony of B after cell fixation and immunostaining. (f) DAPI staining. (g) Merge of DAPI and OCT4 staining. Arrow indicates a MEF whose nucleus does not express OCT4. 
cytoplasm, bright granules within the hESCs and the MEFs are selected by the green, red and blue cluster respectively [Fig. 1(a) and 1(d)]. After label-free FLIM imaging, we fixed the cells and we performed retrospective immunostaining imaging (see Sec. 4) for the phenotypic identification of undifferentiated hESCs and MEFs. Undifferentiated hESCs are identified by the expression of the pluripotency transcription factor OCT4 [Fig. 1(e)-1(g), while MEFs are identified by DAPI staining and the absence of OCT4 expression [arrows in Fig. 1(f) and $1(\mathrm{~g})]$. The specific bright granules within the hESCs are identified by the red cluster in Fig. 1(a) and are homogeneous in dimensions and typically have a diameter of $\sim 1 \mu \mathrm{m}$. [Fig. 2(a) and 2(b)]. They are characterized by a long lifetime distribution [Figs. 1(a)-1(d) and 2(c)] very close to the universal circle (defined in the Appendix) and by a single exponential of about 10 ns. Although the hESC granules have a very similar lifetime to protoporphyrin IX, ${ }^{32}$ we exclude its presence because the hESC granules emission spectrum [Fig. 2(d)] is very different with respect to protoporphyrin IX spectrum that has a peak at $630 \mathrm{~nm} .{ }^{49}$ In fact the emission spectrum of the hESC granules is very broad and it has a peak at $500 \mathrm{~nm}$ [Fig. 2(d)]. The lipiddroplet-associated-granules (LDAG) emission spectrum is similar to the spectrum of flavins. However we exclude presence of flavins both in the granules and in the rest of the hESCs, because we do not detect any fluorescence at 800-, 850-, and 900-nm excitations (Fig. 3).

Figure 4 shows the colocalization of hESC granules with lipid droplets (LDs) labeled in vitro with 4,4-difluoro-1,3,5,7,8pentamethyl-4-bora-3a,4a-diaza-s-indacene (BODIPY493/503). Although granules with similar morphology and which
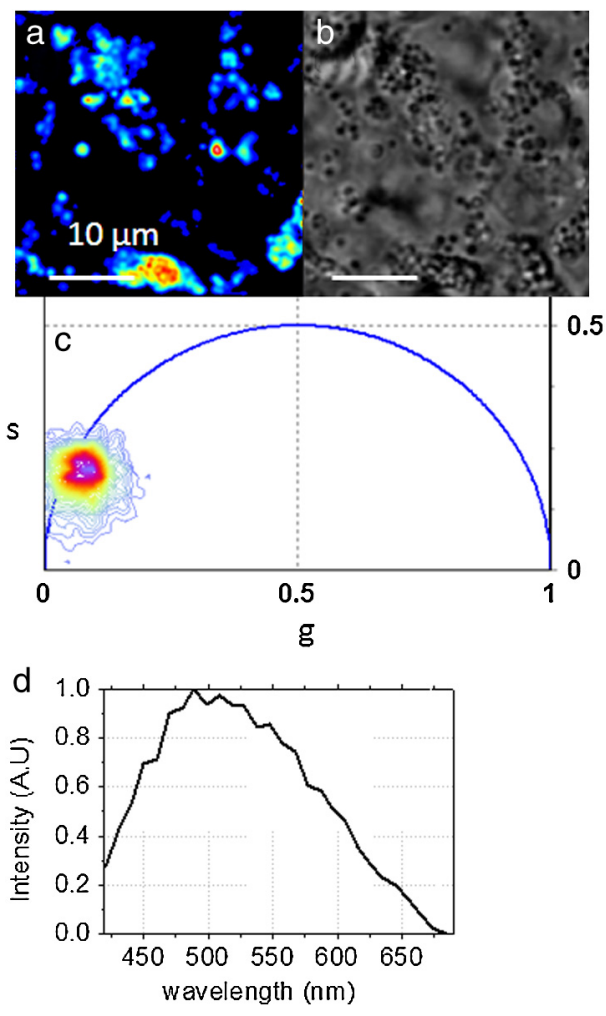

Fig. 2 Spectroscopic characteristics of granules in hESCs: two-photon fluorescence intensity image (a) and transmission image (b) of a single undifferentiated H9 hESCs colony. (c) FLIM phasor plot of the FLIM image excited at $760 \mathrm{~nm}$ of the hESCs colony area in (a). (d) Emission spectrum from hESCs granules in (a).
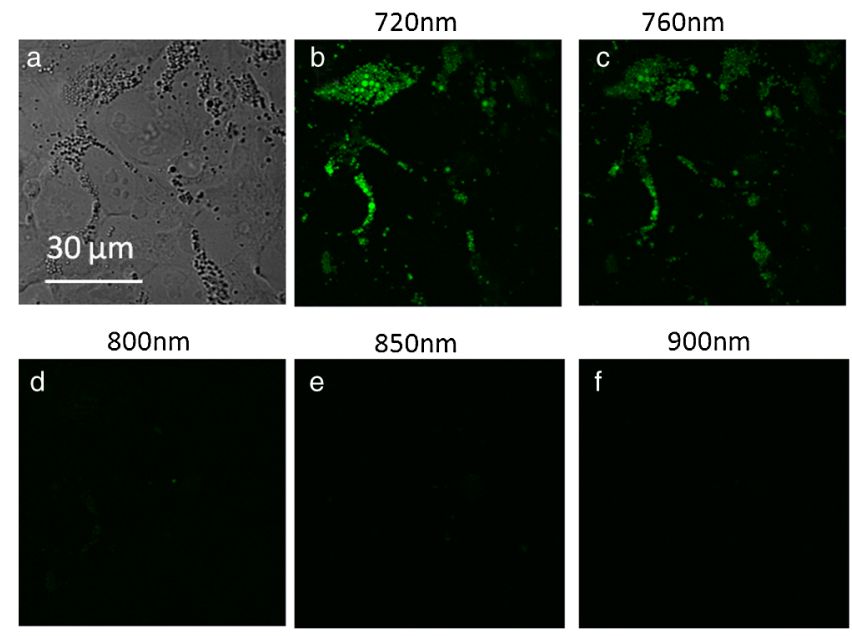

Fig. 3 Transmission image (a) and two-photon fluorescence intensity images of an undifferentiated $\mathrm{H} 9 \mathrm{hESC}$ colony area excited at $720 \mathrm{~nm}$ (b), $760 \mathrm{~nm}$ (c), 800 (d), 850 (e) and 900 (f)

positively stain for lipids have been identifies as lipofuscin in several tissue and cells, ${ }^{28,50}$ we exclude that the LDAG we observe could be related to lipofuscin. LDAG absorption spectrum (Fig. 3) is not as wide as the one of the lipofuscin which strongly fluoresces under excitation ranging from 360 to $660 \mathrm{~nm} .^{28,50}$ The lifetime of the granules that we observe [Fig. 2(c)] is much longer than the lifetime of the lipofuscin reported in the literature. ${ }^{28,51-53}$ Moreover the LDAG do not colocalize with the lysosomes (Fig. 5), which are known to be the structures involved in the storage of lipofuscin ${ }^{54,55}$ and which have been previously colocalized with lipofuscin..$^{30,52,56}$

We hypothesize that the formation of LDAGs in hESC could be generated by the combination of high ROS level, associated to high oxidative-phosphorylation rate $^{46}$ and the abundance of
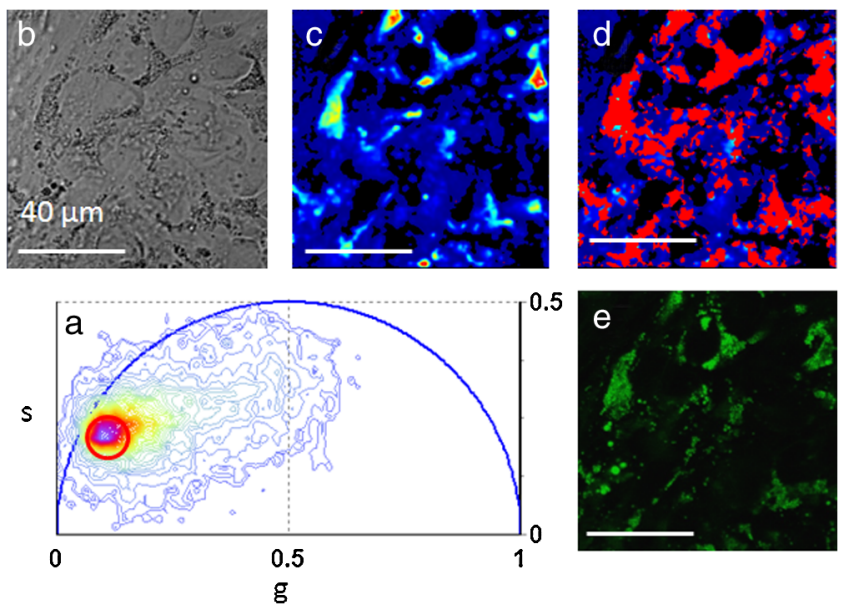

Fig. 4 Colocalization of lipid droplets with granules in hESCs: (a) FLIM phasor plot of the FLIM image excited at $760 \mathrm{~nm}$ of a single undifferentiated H9 hESC colony area. The red cluster in the phasor plot specifically selects some bright granules within the hESCs. Transmission image (b) and two-photon fluorescence intensity image (c) of the hESCs colony area. (d) Phasor color map. Red pixels have a specific lifetime signature that is selected by the red cluster in the phasor plot A. (e) In vitro staining of lipid droplets with Bodipy 493/503 shows colocalization with the hESCs granules identified by FLIM in $\mathrm{D}$ and $\mathrm{A}$. 

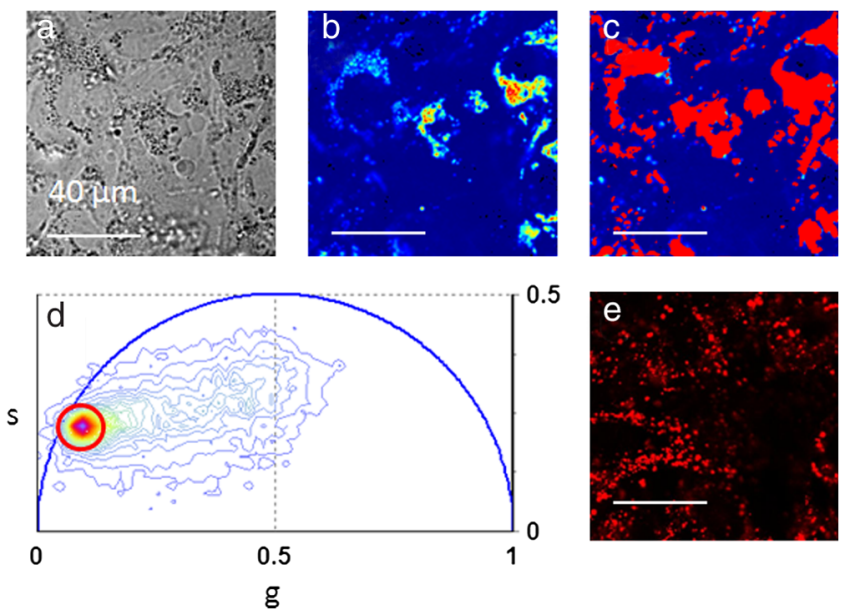

Fig. 5 Nonlocalization of hESC granules with lysosomes: transmission image (a) and two-photon fluorescence-intensity image (b) of an undifferentiated H9 hESC colony area. (c) Phasor color map. hESC granules are highlighted by the red pixels which correspond to the red cluster in the phasor plot D. (d) In vitro FLIM phasor plot of the undifferentiated hESC colony area. The red cluster in the phasor plot specifically selects hESC granules. (e) In vitro staining of lysosomes with Lysotracker.

unsaturated metabolic precursors, such as arachidonic acid, that are highly reactive under oxidative conditions. ${ }^{34}$ Linoleic acid and arachidonic acid peroxides can form fluorescent products from the interaction with polypeptides and free amino acid groups of proteins. ${ }^{57,58}$ LDAGs [Fig. 4(d) and 4(e)] are surrounded by endoplasmic reticulum membranes (Fig. 6). We tested and excluded colocalization between these LDAGs and CONNEXIN-43 (Fig. 7 of the Appendix) and Alkaline phosphatase (Fig. 8 of the Appendix).

To identify the contribution of NADH to the FLIM phasor signature of hESCs, we labeled the mitochondria with tetramethylrhodamine ethyl ester perchlorate (TMRE), a dye-marking active mitochondria (Fig. 9). Our data show that the mitochondria do not specifically localize with the LDAGs, which are indicated by the red clusters; conversely, mitochondria are primarily localized to the blue cluster areas in hESCs at the center of the phasor plot [Fig. 9(a)]. This location corresponds to the typical autofluorescence arising from the NADH within cells and tissues. ${ }^{32}$ ) To confirm the contribution of NADH to the autofluorescence detected in the center of the phasor plot, we treat the hESCs with potassium cyanide (KCN) to block oxidative phosphorylation during cellular respiration and increase the levels of the reduced form of NADH. When hESCs are treated with KCN the FLIM Phasor distribution shifts toward the location of the free reduced NADH [Fig. 10(c)and 10(i)] and the concentration of free NADH increases with respect to bound NADH [Fig. 10(g)and 10(m) of the Appendix].

\subsection{Phasor FLIM Discriminates Undifferentiated hESCs from Differentiating hESC Colonies}

We measured the metabolic signature of hESC differentiation by monitoring the concentrations of the intrinsic fluorescent biomarkers NADH and LDAGs. In Fig. 11 we identified the differences between the FLIM phasor signature of undifferentiated hESCs and differentiating hESCs. To induce differentiation towards trophectoderm or a neurogenic lineage, we cultured hESCs in the presence of BMP4 or retinoic acid (RA) respectively, while simultaneously removing basic fibroblast growth factor (bFGF) from the culture media, which is critical to the maintenance of hESC pluripotency and self-renewal (see Sec. 4) ${ }^{59,60}$ We measure the pluripotency and differentiation of hESC by monitoring the expression of the hESC marker OCT4 [Fig. 11(f) and 11(h)] with retrospective immunostaining after live imaging (see Sec. 4). Undifferentiated hESC have a high expression of OCT4, while differentiating hESC are characterized by lower expression of OCT4 [Fig. 11(f) and 11(h)].

The FLIM phasor distribution of undifferentiated hESCs [Fig. 11(a)], is dominated by the contribution of the hESC granules identified in Figs. 1, 2, and 4. On the other hand, the FLIM phasor distribution of differentiated hESCs treated with BMP4 [Fig. 11(b)], is shifted toward the central region of the phasor plot represented by the FLIM fingerprint of $\mathrm{NADH}^{32}$ (Figs. 9 and 10). During differentiation the lifetime of the LDAGs in hESC does not change, the ratio of free to bound NADH presents a small increase [Fig. 10(f) and 10(1)], and the ratio of LDAG to NADH increases significantly (Fig. 11).

We used a phasor linear cursor [colored bar in Fig. 11(a) and 11(b)] to represent all possible contributions of the LDAGs (purple) and the NADH (cyan-white). Every single color along the line represents a different relative concentration of the two fluorescent metabolic markers. The colored FLIM map of Fig. 11(e) shows that the H9 undifferentiated hESC colony has a higher concentration of LDAGs with respect to NADH, while the differentiating hESC colony has a much lower concentration of LDAGs with respect to NADH.

In Fig. 11(i) and 11(j)we measured the phasor FLIM signature of the entire hESC colony by calculating the average value of its phasor FLIM distribution (see Sec. 4). Figure 11(j) shows that the FLIM signatures of undifferentiated hESCs from two different cell lines, H9 (black squares) and H1 (cyan circles) are localized in the same region of the phasor plot and are not statistically different $(p=0.34)$. The phasor FLIM signatures of undifferentiated H9 hESCs (black squares in Fig. 11(i) are statistically different $(p<0.0001)$ from differentiating H9 hESCs treated with either BMP4 (-bFGF) medium [red circles in Fig. 11(i)], RA (-bFGF) medium [orange triangles in Fig. 11(j)] or -bFGF only medium [purple triangles in Fig. 11(j)]. While the hESCs treated with RA and BMP4 are not statistically distinguishable ( $p=0.25$ ), colonies cultured in the absence of bFGF are statistically different from both conditions $(p<0.0001)$ and have a phasor FLIM signature closer to that of undifferentiated hESCs [Fig. 11(j)].

The trend in Fig. 11(i) and 11(j)of the phasor FLIM signatures of the $\mathrm{H} 9$ colonies during differentiation reflects differences in relative concentration of LDAGs and NADH, that is mapped in Fig. 11(e). The increase of NADH concentration (Fig. 11) and the small increase in free-to-bound $\mathrm{NADH}$ [Fig. 10(f) and 10(1)] during early hESC differentiation is in agreement with the decrease of oxidative-phosphorylation rate with respect to glycolysis during early hESC differentiation. ${ }^{46}$ We hypothesize that the abundance of LDAGs in undifferentiated hESCs indicates the formation of fluorescent aggregates generated by the interaction of ROS and unsaturated lipids. Hence the decrease in LDAGs concentration during differentiation can reflect a decrease in the abundance of unsaturated eicosanoids, such as linoleic acid and arachidonic acid, that promote pluripotency, ${ }^{34}$ the activation of oxidation and a decrease in antioxidant enzymes concentration ${ }^{36,61}$ or a decrease in ROS level following a decrease in oxidative-phosphorylation rate. $^{46}$ 

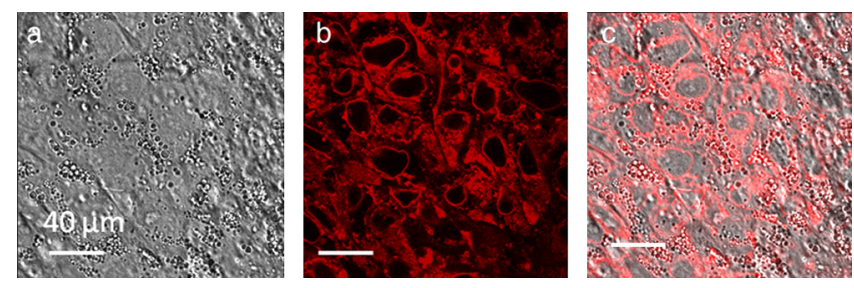

Fig. $6 \mathrm{hESC}$ granules are surrounded by endoplasmic reticulum: (a) transmission image of an undifferentiated H9 hESC colony area. (b) In vitro staining of endoplasmic reticulum with ER-Tracker Red. (c) Merge of transmission image and ER staining.

\subsection{Cell Phasor FLIM of Heterogeneity in hESCS}

The phasor FLIM analysis at single cell resolution reveals heterogeneity in the metabolic signature and intrinsic biomarker content of hESCs within the same colony. We exploit the cell-phasor concept and image segmentation (Ref. 32 and Sec. 4) to measure the phasor FLIM signature of single hESCs by calculating the average value of the phasor distribution of single hESCs (see Sec. 4).

Figure 12(a) and 12(b) show the plot of single hESCs from an undifferentiated H9 colony (black dots), an undifferentiated H1 colony (cyan dots) and a differentiating H9 colony treated with BMP4 (red dots). The cell phasor cluster of undifferentiated $\mathrm{H} 9 \mathrm{hESCs}$ is smaller in size compared to the cell phasor cluster from differentiating H9 hESCs [Fig. 12(a)] and the standard deviation of the g component (defined in the Appendix) of the cell phasors from an undifferentiated H9 colony is smaller
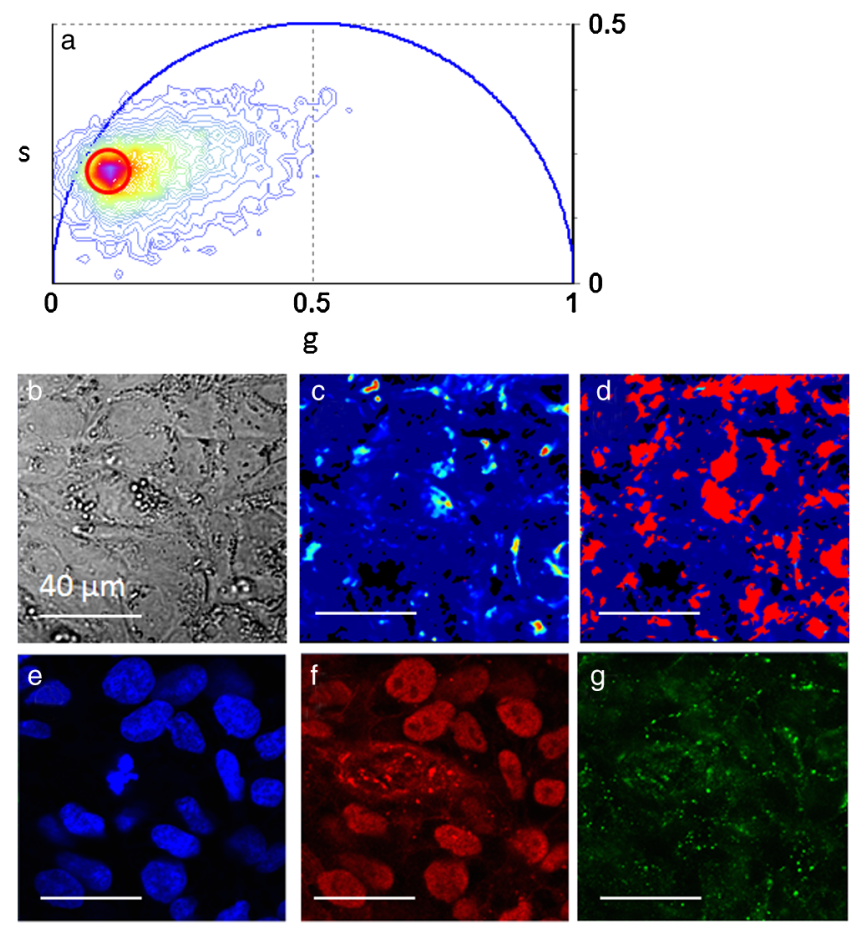

Fig. 7 Nonlocalization of hESC granules with CONNEXIN-43: (a) In vitro FLIM phasor plot of an undifferentiated H9 hESC colony. The red cluster selects the bright granules within hESCs. Transmission image (b) and two-photon fluorescence intensity image (c) of the undifferentiated hESC colony. (d) Phasor color map. hESC granules are highlighted by the red pixels that correspond to the red cluster in the phasor plot A. (e) DAPI staining. Expression of the pluripotency marker OCT4 (f) and CONNEXIN-43 (g) after cell fixation and immunostaining.
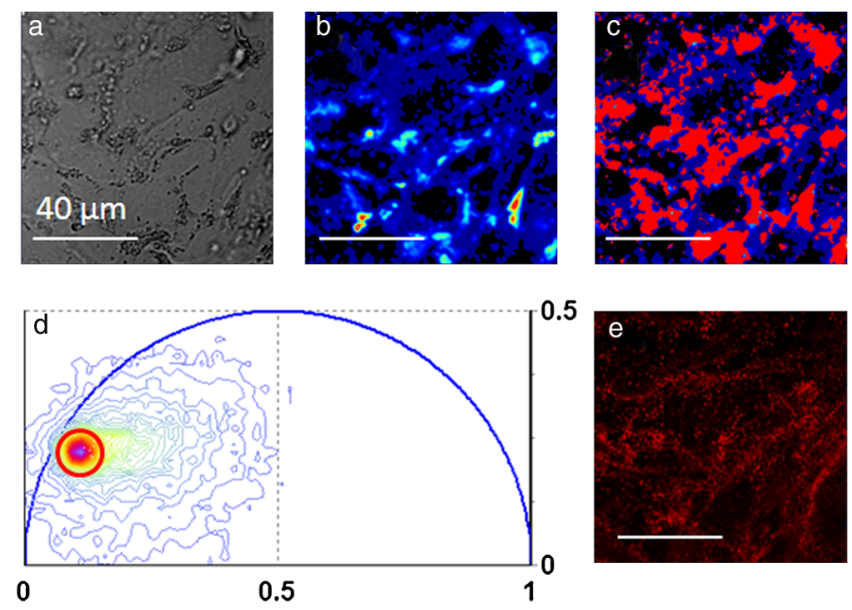

Fig. 8 Nonlocalization of hESC granules with alkaline phosphatase: transmission image (a) and two-photon fluorescence intensity image (b) of an undifferentiated H9 hESC colony area. (c) In vitro FLIM phasor plot of an undifferentiated hESC colony area. The red cluster specifically selects hESC granules. (d) Phasor color map. hESC granules are highlighted by the red pixels that correspond to the red cluster in the phasor plot C. (e) Alkaline phosphatase staining performed after cell fixation.

than that of differentiating H9 hESCs [Fig. 12(c)]. This observation indicates that the FLIM signature of single hESCs in a H9 colony becomes more heterogeneous during differentiation. Cell phasor and FLIM signature of single hESCs is determined by the relative concentration of the intrinsic fluorescent metabolites NADH and LDAGs (Figs. 2, 4, and 9). The cell phasor reveals the metabolic signature of the cells that might be associated with the stem cells' phenotype and be indicative of commitments to different differentiation pathways. The higher degree of cell phasor heterogeneity within the $\mathrm{H} 1$ hESC colonies [Fig. 12(c)] might reflect the higher phenotypic heterogeneity and differential expression of hESC markers that are known to be present in H1 hESC-lines. ${ }^{62}$
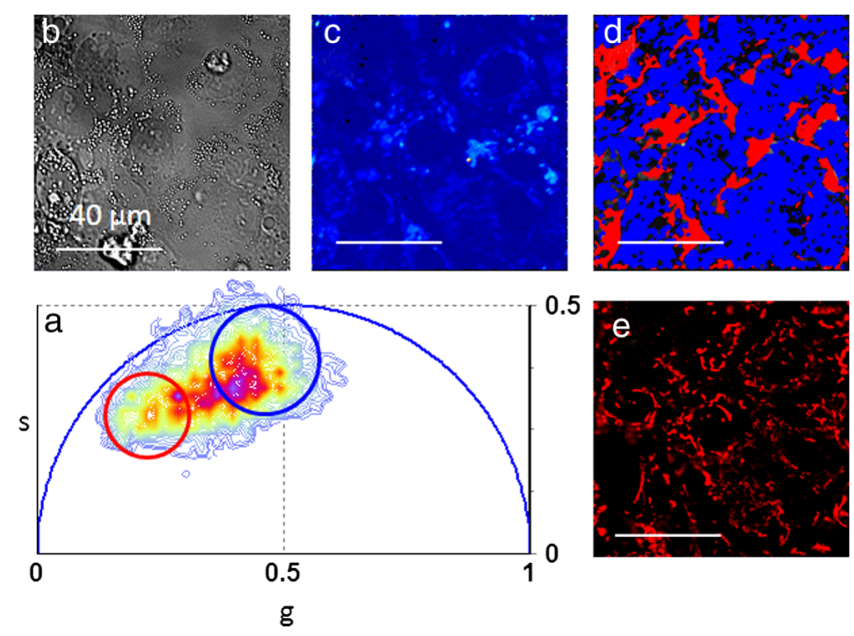

Fig. 9 Mitochondria colocalization and contribution of NADH: (a) FLIM phasor plot of the FLIM image excited at $760 \mathrm{~nm}$ of a single differentiating H9 hESC colony area. The red cluster in the phasor plot specifically selects some bright granules within the hESCs, while the blue cluster selects the rest of the cellular cytoplasm and cell nuclei. Transmission image (b) and two-photon fluorescence intensity image (c) of the hESC colony area. (d) Phasor color map of the hESC colony area. Pixels of different colors correspond to the color of the cluster in the phasor plot (a). (e) In vitro staining of mitochondria with TMRE. 


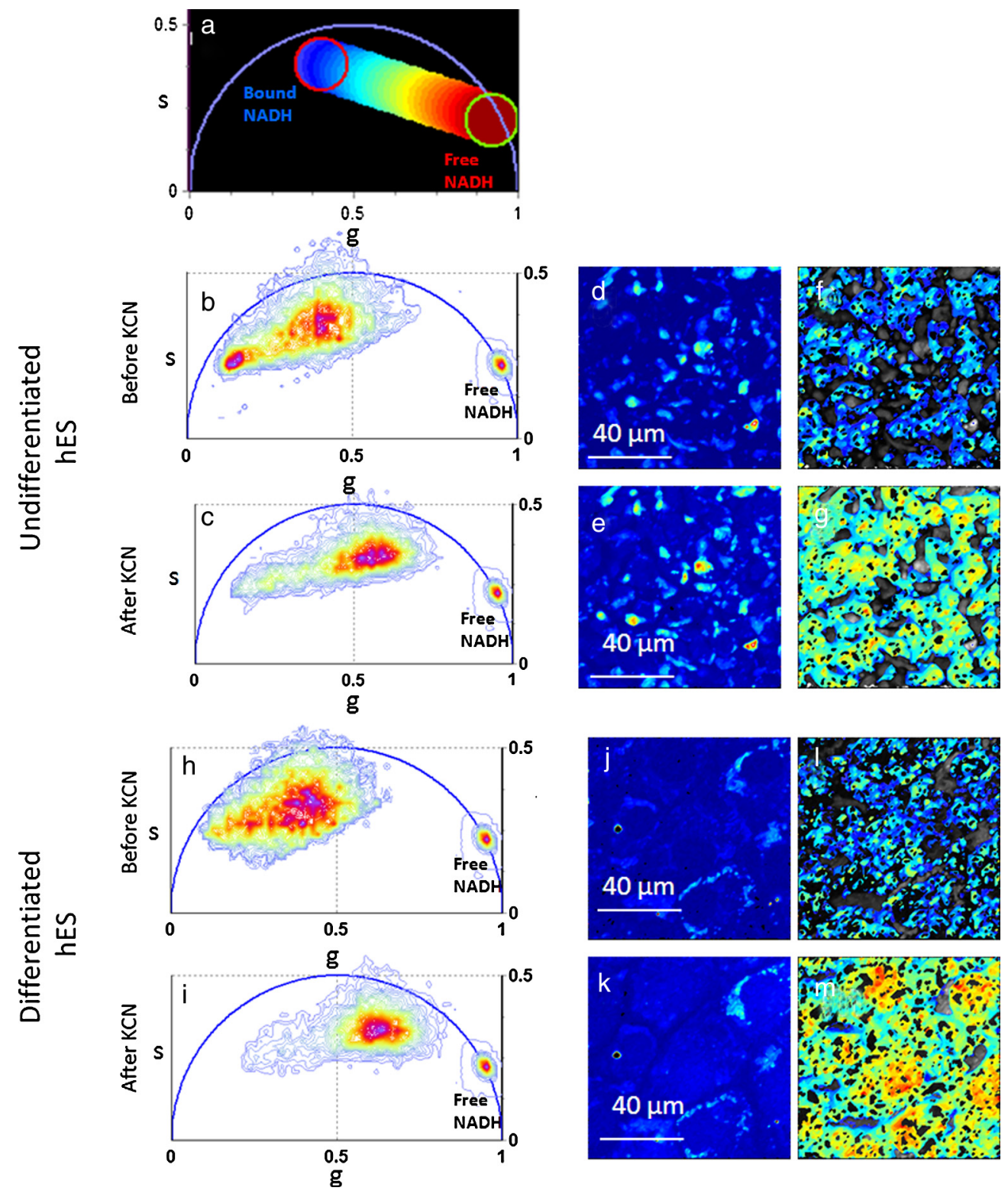

Fig. 10 Effect of electron-transport-chain inhibition on the hESC FLIM phasor distribution: (a) Phasor plot selection using linear cluster combination that represents all the possible relative concentrations of bound NADH and freeNADH. The phasor locations of pure boundand freeNADH have been measured in Ref. 32. Each point along the line has a color corresponding to specific relative concentration of bound-to-free NADH. In vitro FLIM phasor plot of an undifferentiated $\mathrm{H} 9 \mathrm{hESC}$ colony $(\mathrm{b}, \mathrm{c})$ and a differentiating $\mathrm{H} 9 \mathrm{hESC}$ colony $(\mathrm{h}, \mathrm{i})$ before and after the treatment with potassium cyanide (KCN). TwO-photon fluorescence-intensity images of the undifferentiated $\mathrm{hESC}$ colony $(\mathrm{d}-\mathrm{e}$ ) and differentiating hESC colony $(\mathrm{j}-\mathrm{k}$ ) before and after the KCN treatment. Phasor color map images representing the relative concentrations of bound and free NADH in the undifferentiated hESC colony $(\mathrm{f}-\mathrm{g}$ ) and the differentiating hESC colony $(\mathrm{I}-\mathrm{m})$ before and after the $\mathrm{KCN}$ treatment. By blocking the respiratory chain in $\mathrm{hESC}$, the FLIM phasor distribution shifts toward the location of the free reduced $\mathrm{NADH}(\mathrm{c}, \mathrm{i})$ and the cell concentration of free $\mathrm{NADH}$ increases with respect to bound $\mathrm{NADH}(\mathrm{g}, \mathrm{m})$.

Moreover, Fig. 12(c) shows that the cell phasor cluster size of both undifferentiated and differentiating hESC colonies are smaller than the size of the MEF cluster [green dots in Fig. 12(b)]. The heterogeneity in the FLIM/metabolic signatures of hESCs might reflect different cell phenotypes and plasticity that are reduced in a defined fully differentiated cell types such as MEFs.

\section{Discussion}

In this work we demonstrate that we can detect the level of embryonic-stem-cell metabolic activity and differentiation by phasor analysis and FLIM. Our method is label-free and completely noninvasive, thus maintaining cell viability for in vitro studies and clinical transplantation.
We identify two intrinsic metabolic biomarkers that allow us to discern the differentiation state of hESCs. These fluorescent intrinsic biomarkers are NADH and LDAGs that display unique fluorescent lifetime properties (Figs. 1, 2, and 9). We believe that these LDAGs are peculiar to embryonic stem cells. Their presence in hESC could be generated by the combination of high ROS level, associated to high oxidative-phosphorylation rate $^{46}$ and the abundance of unsaturated metabolic precursor, such as arachidonic acid, that are highly reactive under oxidative conditions. ${ }^{34}$ In the previous literature there is no evidence of granules with similar spectroscopic characteristics associated with adult stem cell. ${ }^{29-31,56}$ We exclude any relation of LDAGs with lipofuscin because of their different absorption 

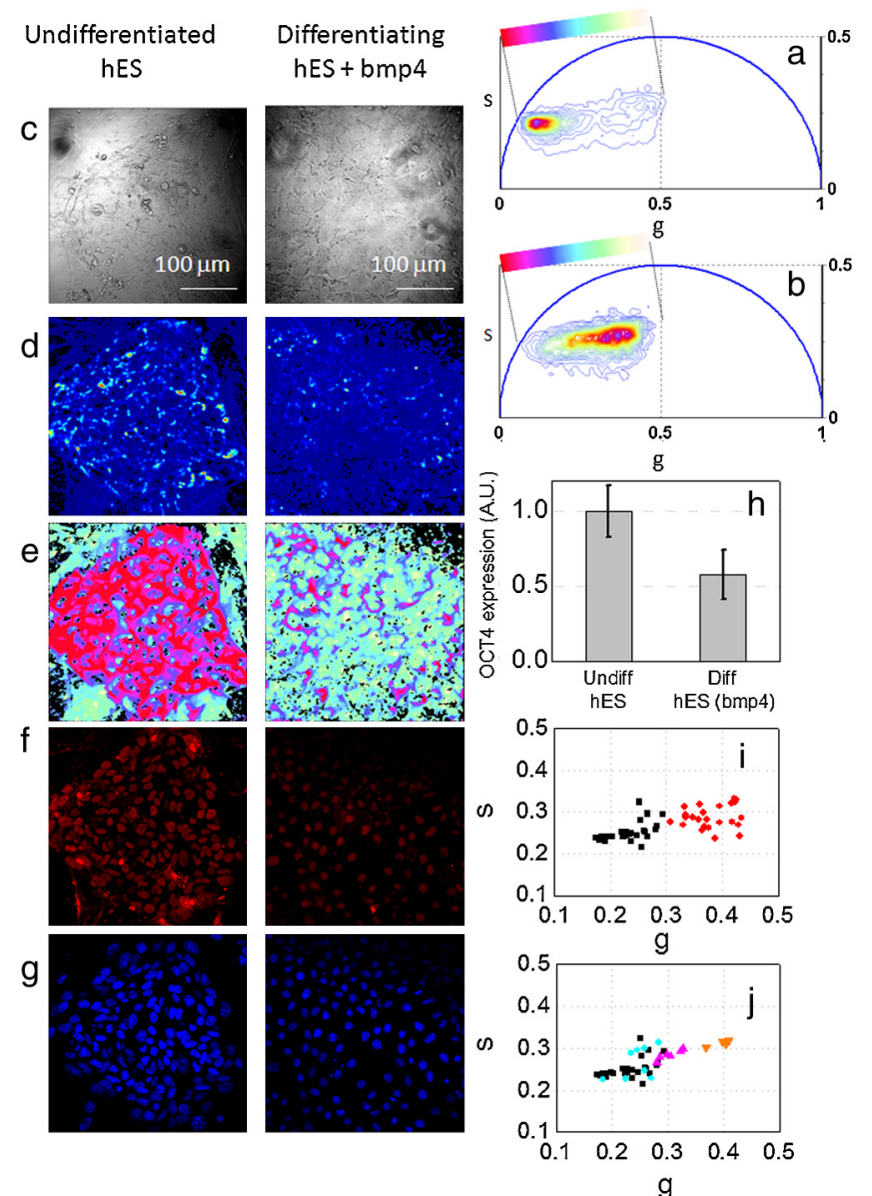

Fig. 11 FLIM phasor separates undifferentiated from differentiated hESC colonies: FLIM phasor plot of the FLIM image excited at $760 \mathrm{~nm}$ of a single undifferentiated $\mathrm{H} 9 \mathrm{hESC}$ colony area (a) and a differentiating $\mathrm{H} 9$ hESC colony treated with BMP4 medium for four days (b). Phasor plot selection using linear cluster combination that represents all the possible relative contributions of the hESCs granule FLIM signature (purple) identified in Figs. 1 and 2 and the NADH FLIM signature typical of the MEFs (cyan-white), identified in Figs. 1 and 4. Each point along the line has a color that corresponds to a specific relative concentration of the two species. Transmission images (c) and two-photon fluorescence intensity images (d) of the undifferentiated hESC colony and the differentiating hESC colony. (e) Phasor color map images representing the relative concentration hESC granules (purple) and NADH (cyanwhite) according to the color scale in Fig. A and B. (f) Expression of the pluripotency marker OCT4. (g) DAPI staining. (h) Oct4 expression in undifferentiated hESC compared to differentiating hES cells with BMP4 medium for four days, measured from image (f). (i)-(j) Scatter plot of the phasor FLIM signature of hESC colonies. Every point represents the average phasor value of an entire hESC colony. Black and cyan squares represent undifferentiated $\mathrm{H} 9$ colonies $(N=27)$ and undifferentiated $\mathrm{H} 1$ colonies respectively $(N=8)$. Red circles represent differentiating H9 colonies treated with BMP4 media for four days $(N=24)$, purple triangles $\mathrm{H} 9 \mathrm{hESC}$ colonies differentiating in a medium without bFGF $(N=6)$, orange triangles $\mathrm{H} 9 \mathrm{hESC}$ s induced to differentiate in RA medium for four days $(N=6)$.

characteristics (Fig. 3) and lifetime signature (Fig. 2) and the lack of localization of LDAG with lysosomes. ${ }^{28,30,51-53}$

The differential change in the concentration of LDAG and NADH provides a clear FLIM signature of undifferentiated and differentiating hESCs cells (Figs. 11 and 12). We map the relative concentration of NADH and LDAGs in hESC colonies (Fig. 11) and distinguish the phasor FLIM
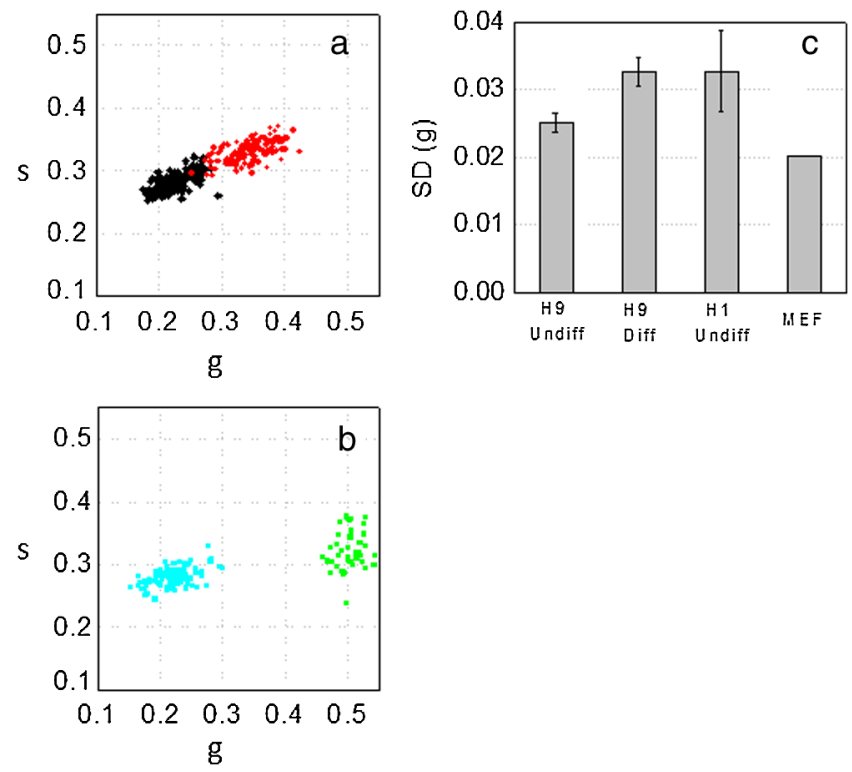

Fig. 12 Heterogeneity within hESC colonies: (a) Scatter plot of the phasor FLIM signature of individual hESCs from the two colonies displayed in Fig. 11. Every point represents the average phasor value of a single hES cell. Black squares represent undifferentiated H9 hESCs $\left(N_{\text {cells }}=123\right)$ and red circles represent differentiating H9 hESCs treated with BMB4 media for four days $\left(N_{\text {cells }}=136\right)$. (b) Cyan squares represent the cell phasor of $\mathrm{H} 1$ hESCs $\left(N_{\text {cells }}=119\right)$ from the colony and green squares represent MEFs $\left(N_{\text {cells }}=42\right)$. (c) Standard deviations $\left(N_{\text {colony }}=3\right)$ of the phasor g coordinates of the cell phasor of single hESCs from an undifferentiated $\mathrm{H} 9$ colony, differentiating $\mathrm{H} 9$ and $\mathrm{H} 1$ colony and MEFs.

signature of undifferentiated hESCs from differentiated hESCs using common differentiation protocols (Fig. 11). We demonstrated that the phasor FLIM signature depends on the level of hESC differentiation [Fig. 11(i) and 11(j)] and correlates with the level of expression of the pluripotency marker OCT4 [Fig. 11(e), 11(f), and 11(h)].

We believe the differentiation-induced change in the intrinsic FLIM signature of hESCs is related to a modulation of endogenous metabolites involved in oxidative pathways that mediate stem-cell differentiation. During early hESC differentiation we measured an increase of NADH concentration (Fig. 11) and we observed a small increase in the free-to-bound NADH ratio [Fig. 10(f) and 10(1)]. Our findings are in agreement with the lower rate of oxidative phosphorylation that Birket et al. ${ }^{46}$ measured in differentiating hESCs compared to undifferentiated hESCs. While our results suggest that oxidative phosphorylation rate decreases during early hESC differentiation with respect to glycolysis, the opposite trend has been shown during differentiation of adult stem cells. ${ }^{29,30,46}$ In the case of adipogenic differentiation, Koenig et al. ${ }^{30}$ showed a decrease of NADH fluorescence intensity and a decrease of free-tobound NADH during differentiation and Guo et al. ${ }^{29}$ reported an increase of NADH lifetime during osteogenic differentiation.

Our findings indicate that the metabolic changes occurring during early differentiation of hESC are different with respect to the advanced stages of differentiation of adult stem cells into completely differentiated cells. Consistently Birket et al. ${ }^{46}$ showed that the transition from pluripotent stem cells through progressive stages of differentiation involves dynamic changes in the energy demand and in relative contributions of the oxidative and glycolytic metabolic pathways. 
The hESC granules that colocalize with lipid droplets and have a specifically long lifetime (Figs. 1, 2, and 4) are highly expressed in the undifferentiated hESCs, and their concentration decreases significantly upon hESC differentiation (Fig. 11). We believe that these LDAGs are related to the level of ROS and of metabolic oxidation activity in the cells. hESCs are in fact characterized by a high ATP turnover and mitochondrial activity that produce a high level of ROS. ${ }^{46}$ We propose that the high number of LDAGs in undifferentiated hESCs might contain aggregates of fluorescent lipid peroxidation-modified proteins generated by the action of ROS. ${ }^{57,58}$ These granules could be correlated to the abundance of structurally unsaturated metabolites, and specific endogenous inflammatory mediators, such as eicosanoids, that regulate the regenerative properties of stem cells and whose levels decrease upon differentiation. ${ }^{34}$ The high levels of linoleic acid, arachidonic acid, eicosapentaenoic acid and docosahexaenoic acid ${ }^{34}$ and the high level of ROS ${ }^{46}$ in undifferentiated hESCs can lead to the formation of fluorescent aggregates. ${ }^{57,58}$ Our results are in agreement with the observation by Birket et al. ${ }^{46}$ that important antioxidant-proteins levels do not significantly change during early hESC differentiation, regardless of the rate of oxidative phosphorylation.

We demonstrated that the FLIM signature of single hESCs reveals a higher heterogeneity in the metabolic state of differentiating hESCs (Fig. 12). This might reflect phenotypic heterogeneity within the individual stem cell colonies. Although the FLIM signature of undifferentiated H9 and H1 hESC colonies are very similar [Fig. 11(j)], a cell phasor analysis reveals higher FLIM-metabolic heterogeneity within H1 hESC colonies.

FLIM and emission spectroscopy have previously been used to discriminate in vitro human mesenchymal stem cells ${ }^{29,56}$ and human salivary gland stem cells ${ }^{30,31}$ from differentiated progenies. To our knowledge this is the first time FLIM has been used to study hESCs.

Phasor FLIM not only has the capability to discriminate different metabolic states of hESCs associated with differentiation (Figs. 11 and 12), but also has the potential to predict stem-cell fate and the commitment to different cell lineages. Stem cells with different metabolic rates can be undergoing different fate decisions, but based on morphology and pluripotencymarker expression are indistinguishable from one another. ${ }^{63}$ Relatively small changes in intracellular metabolite levels can have profound influences over cell fate decisions and cellular functions. ${ }^{40,64,65}$ Hence by measuring the metabolic activity and redox ratio of cells, it might be possible to predict the commitment of stem cells to different differentiation pathways, independent of the expression of pluripotency and lineagemarker-expression profiles.

Phasor FLIM is a promising label-free and noninvasive tool that provides metabolic signatures of hESCs in vitro and has the ability to distinguish hESC states (Fig. 11). FLIM enables in vitro monitoring of hESC metabolic activity, heterogeneity, plasticity and stability, indicators which can be used for isolating cells for transplantation and tissue engineering. This technique is very suitable for cell sorting because it is not destructive and does not require exogenous markers or cell treatments that can compromise cell viability. Phasor FLIM represents a powerful method for biophotonics, stem-cell biology and regenerative medicine as well as a new platform for cell sorting, high content analysis, metabolomics and drug screening.

\section{Materials and Methods}

\subsection{Cell Culture}

We used federally approved H9 and H1 human embryonic stem cell lines. hESCs are cultured on a substrate of mouse fibroblast (MEF) feeders (Chemicon Cat \#PMEF-CF). Plates are first coated with $0.1 \%$ to $0.2 \%$ gelatin (Sigma \#G-1393). MEFs are then plated with a density of approximately of 15,000 cells per $\mathrm{cm}^{2}$. hESCs ranging from passage 42 to 57 were used for image analysis. hESCs were grown in hESC cell lines $\mathrm{H} 1$ and $\mathrm{H} 9$ and were cultured in a medium containing: DMEM-F12 (Invitrogen 12660), 20\% KnockOut serum replacement (KOSR, Invitrogen 10828), $1 \%$ nonessential amino acids (NEAA, Invitrogen 11140-050), 1 mM Glutamax (Invitrogen 35035), $4 \mathrm{ng} / \mathrm{mL}$ basic fibroblast growth factors (bFGF, Invitrogen 13256-029) and $0.1 \mathrm{mM}$ beta-mercaptoethanol, as described by $\mathrm{Xu}$ et al. ${ }^{66}$ Medium was changed every day and hESC were maintained in an incubator at $37 \mathrm{uC}$ with $5 \%$ $\mathrm{CO} 2$. Differentiation was induced by removing bFGF from hESC medium and adding either $100 \mathrm{ng} / \mathrm{ml}$ BMP4 (R\&D) or RA $(1 \mu \mathrm{M})$ as described by Pera et al. ${ }^{67}$ We image hESC during their early differentiation stages, after four days of treatment with BMP4 or RA. Treating embryonic stem cells with BMP4 differentiates them towards trophectoderm, while RA differentiates them down the neurogenic lineage. ${ }^{59}$

\subsection{MEFs Medium}

Dulbecco's Modified Eagle Medium (DMEM), 1 mM Glutamax (Invitrogen 10569), 10\% fetal bovine serum (FBS, Invitrogen 16000-044) and 1\% nonessential amino acids (Invitrogen 11149-035) were used.

\subsection{In Vitro Staining}

Lipid droplets were stained with BODIPY 493/503 (Invitrogen \#D3922). hESCs mitochondria were stained with $1 \mathrm{ul} / 1 \mathrm{ml}$ TMRE (Sigma \#87917, excitation/emission: 540/595 nm) solution. After $30 \mathrm{~min}$ of staining cells were washed and imaged. hESCs lysosomes were stained with $50 \mathrm{nM}$ LysoTracker Red (Invitrogen L7528, excitation/emission: 577/590 nm) in phosphate buffered saline (PBS). Endoplasmic reticulum was stained with ER-Tracker ${ }^{\mathrm{TM}}$ Red (Invitrogen \#E34250).

\subsection{KCN Treatment}

We block the respiratory chain by means of $\mathrm{KCN}$ to inhibit the oxidative phosphorylation and increase the mitochondrial concentration of NADH. KCN in PBS was added to the culture medium with a final concentration of $4 \mathrm{mM}$. Cells were imaged immediately after the addition of KCN.

\subsection{Alkaline Phosphatase Staining}

Once the media are removed, cells are washed with PBS minus and fixed for 20 min using $4 \%$ paraformaldehyde (PFA). Cells were washed three times with deionized water. $1 \mathrm{mg} / \mathrm{ml}$ of FastRed is dissolved in deionized water right before use, then combined with $40 \mu \mathrm{l} / \mathrm{ml}$ of Napthol. hESCs were stained for $20 \mathrm{~min}$ and then washed with deionized water. 


\subsection{Immunostaining}

Medium was removed from the stem-cell plate and washed with PBS. Cells were fixed in 4\% PFA. hESCs were washed three times with PBS, permeabilized with $1 \mathrm{ml}$ cold Methanol for $5 \mathrm{~min}$ at room temperature and then washed three times with PBS. The cells were blocked for an hour at room temperature using 10\% Donkey serum (Sigma D9663) in PBS. Primary antibodies OCT4 (R\&D AF1759-1:100) and CONNEXIN-43 (Cell Signaling 3512-1:50) were diluted in 1\% Donkey serum and incubated with the cells for $1 \mathrm{hr}$ at room temperature. Cells were washed three times with PBS. The secondary antibody Donkey anti-Goat Alexa 568 (Invitrogen A-11057) was used at a 1:400 and incubated with the hESCs for $1 \mathrm{~h}$ at room temperature. DAPI solution was added to the cell to stain DNA. Finally cells were washed with PBS.

\subsection{Confocal Imaging}

DAPI was excited with 405-nm laser. TMRE, LysoTracker Red, ER-Tracker Red and anti-Goat Alexa 568 were excited with the 561-nm laser. BODIPY 493/503 was excited with the 488-nm laser.

\subsection{Multiphoton and Fluorescence Lifetime Imaging}

Fluorescence lifetime images were acquired with a Zeiss 710 microscope coupled to a Ti:Sapphire laser system (SpectraPhysics Mai Tai) and an ISS A320 FastFLIM. ${ }^{47}$ A $40 \times 1.2$ NA water immersion objective (Zeiss Korr C-Apochromat) was used. For image acquisition the following settings were used: image size of $256 \times 256$ pixels and scan speed of $25 \mu \mathrm{s} /$ pixel. A dichroic filter $(690 \mathrm{~nm})$ was used to separate the fluorescence signal from the laser light and the fluorescence. For the acquisition of FLIM images, fluorescence was detected by a photomultiplier (H7422P-40 of Hamamatsu) and a 610-nm short-pass filter was placed in front of the detector. FLIM data were acquired and processed by the SimFCS software developed at the Laboratory of Fluorescence Dynamics (LFD), University of California Irvine. The excitation wavelength was $760 \mathrm{~nm}$ if not differently specified. An average power of about $5 \mathrm{~mW}$ was used to excite the live hESCs. FLIM calibration of the system was performed by measuring the known lifetime of the fluorescein with a single exponential of 4.04 ns. FLIM data were collected until 100 counts in the brightest pixel of the image were acquired. Typically the acquisition time was less than $1 \mathrm{~min}$. We verified that the autofluorescence of all MEF and hESC culture media does not interfere with the FLIM and spectral signature of cellular autofluorescence. We verified that the FLIM signature of undifferentiated hESC does not change with number of cell passage and size of the colony.

\subsection{FLIM Data Analysis}

Every pixel of the FLIM image was transformed in one pixel in the phasor plot as previously described and reported ${ }^{32,48}$ in detail in the Appendix. The coordinates $g$ and $s$ in the phasor plot were calculated from the fluorescence-intensity decay of each pixel of the image by using the transformations defined in the Appendix. The analysis of the phasor distribution was performed by cluster identification. Clusters of pixel values were detected in specific regions of the phasor plot. Fractional intensities of chemical species in every pixel of the image were evaluated with a graphical analysis in the phasor plot (see Appendix). We performed image segmentation on the FLIM data by selecting the region of interest within the sample. The region of interest of single hESCs or hESC colonies was selected by using a cursor of arbitrary shape. We calculate the phasor average value within these regions of interest. When measuring the cell phasor, all pixels of the cell (about 1000) were taken in account and the signal-tonoise ratio of the FLIM signature of cells is higher than in single pixels. All phasor transformation and the data analysis of FLIM data are performed using SimFCS software developed at the LFD.

\section{Acknowledgments}

This work was supported by NIH-P41 P41-RRO3155, P50GM076516, NIH RO1, HD49488, NIH PO1 HD47675, CIRM RC1-00110 to PD.

The authors would like to acknowledge the helpful comments from Hongtao Chen and Michelle Digman. The authors would like to acknowledge Anna Pensalfini and Charles G. Glabe for providing tissues of old human brains in which we measured the lifetime of lipofuscin. The authors would also like to thank Diep Nguyen and Michelle Khine for providing a dish of H9 undifferentiated hESCs that has been used to confirm the FLIM signature of human embryonic stem cells.

\section{Appendix: Phasor Transformation and Resolution of a Mixture of Components}

When fluorescence lifetime data are acquired in the time domain, the components $\mathrm{g}(x$-coordinate) and $s$ ( $y$-coordinate) of the phasor plot are given by the following expressions:

$$
\begin{aligned}
g_{i, j}(\omega) & =\frac{\int_{0}^{\infty} I_{i, j}(t) \cos (\omega t) \mathrm{dt}}{\int_{0}^{\infty} I_{i, j}(t) \mathrm{dt}}, \\
s_{i, j}(\omega) & =\frac{\int_{0}^{\infty} I_{i, j}(t) \sin (\omega t) \mathrm{dt}}{\int_{0}^{\infty} I_{i, j}(t) \mathrm{dt}},
\end{aligned}
$$

where the indices $i$ and $j$ identify a pixel of the image and $\omega$ frequency $(\omega=2 \pi f)$, where $f$ is the laser repetition rate, i.e., $80 \mathrm{MHz}$ in our experiment. All phasor plots are calculated at $80 \mathrm{MHz}$, i.e., the first harmonic of the laser-repetition rate and in some cases for higher harmonics.

The phasor transformations of FLIM data acquired in the frequency domain at an angular modulation frequency $\omega$ are:

$$
\begin{aligned}
& g_{i, j}(\omega)=m_{i, j} \cos \varphi_{i, j}, \\
& s_{i, j}(\omega)=m_{i, j} \cos \varphi_{i, j},
\end{aligned}
$$

where $m_{i, j}$ and $\varphi_{i, j}$ are the modulation and the phase of the emission with respect to the excitation. Estimations of the lifetime in terms of the phase and modulation can be performed in each pixel by the following formulas:

$$
\begin{gathered}
\tau_{\varphi}=\frac{1}{\omega} \tan (\varphi) \\
\tau_{m}=\frac{1}{\omega} \sqrt{\left(\frac{1}{m^{2}}-1\right) .}
\end{gathered}
$$

In the case of a single exponential decay the two lifetimes obtained by the phase and by the modulation with Eqs. (5) 
and (6) are equal, while for a multiexponential lifetime system the apparent lifetimes are different.

In the phasor plot if the decay is a single exponential $I(t)=$ $A e^{-t / \tau}$ the coordinates are given by:

$$
\begin{aligned}
& g(\omega)=\frac{1}{1+(\omega \tau)^{2}}, \\
& s(\omega)=\frac{1}{1+(\omega \tau)^{2}},
\end{aligned}
$$

where $\tau$ is the lifetime of the decay and $\omega$ is the laser frequency. There is a direct relationship between a phasor location and lifetime. Every possible lifetime can be mapped into this universal representation of the decay (phasor plot). All possible single exponential lifetimes lie on the "universal circle" defined as the semicircle going from point $(0,0)$ to point $(1,0)$ with radius $1 / 2$. Point $(1,0)$ corresponds to $\tau=0$, while point $(0,0)$ to $\tau=\infty$. In the phasor coordinates the single lifetime components add directly because the phasor follows the vector algebra. A mixture of two distinct single lifetime components, each of which lie separately on the single lifetime semicircle, does not lie on the semicircle. All the combination of two single exponential components must be along the line joining the two lifetime points. In a system with many single lifetime components the phasor coordinates $\mathrm{g}$ and $\mathrm{s}$ are described as:

$$
\begin{aligned}
& g(\omega)=\sum_{k} \frac{h_{k}}{1+\left(\omega \tau_{k}\right)^{2}}, \\
& s(\omega)=\sum_{k} \frac{h_{k} \omega \tau_{k}}{1+\left(\omega \tau_{k}\right)^{2}},
\end{aligned}
$$

where $h_{k}$ is the intensity-weighted fractional contribution of the single-exponential component with lifetime $\tau_{k}$. The phasor location of the mixture of single lifetimes is the intensity-weighted average of the contributions of each single lifetime that lie separately on the semicircle.

In general, in a system with multiple fluorescent components like a tissue, the overall decay is a phasor that is the sum of the independent phasors of each fluorescence component:

$$
\begin{aligned}
& G(\omega)=\sum_{n} f_{n} g_{n}(\omega), \\
& S(\omega)=\sum_{n} f_{n} s_{n}(\omega),
\end{aligned}
$$

where $f_{n}$ is the fractional contribution of each component characterized by the phasor coordinates $g_{n}$ and $s_{n}$. Two molecular species with multiexponential decay are identified by two specific points in the phasor plot inside the semicircle. All possible weighting of the two molecular species give phasors distributed along a straight line joining the phasors of the two species. In the case of three molecular species, all the possible combinations are contained in a triangle where the vertices correspond to the phasor of the pure species. The phasor plot of an $N$ component mixture will be contained in a polygon with $N$ vertices located in the position of the phasor of each contributing component. The calculation of the fractional intensities of different fluorescence components that contribute to the signal is performed by a linear estimation on the system described by Eqs. (11) and (12) by graphically resolving the sum of phasors.

\section{References}

1. P. J. Donovan and J. Gearhart, "The end of the beginning for pluripotent stem cells," Nature 414(6859), 92-97 (2001).

2. K. Takahashi et al., "Induction of pluripotent stem cells from adult human fibroblasts by defined factors," Cell 131(5), 861-872 (2007).

3. J. A. Thomson et al., "Embryonic stem cell lines derived from human blastocysts," Science 282(5391), 1145-1147 (1998).

4. J. Yu et al., "Induced pluripotent stem cell lines derived from human somatic cells," Science 318(5858), 1917-1920 (2007).

5. T. Enver et al., "Stem cell states, fates, and the rules of attraction," Cell Stem Cell 4(5), 387-397 (2009).

6. M. H. Stewart et al., "Clonal tracking of hESCs reveals differential contribution to functional assays," Nat. Methods 7(11), 917-922 (2010).

7. C. T. Carson, S. Aigner, and F. H. Gage, "Stem cells: the good, bad and barely in control," Nat. Med. 12(11), 1237-1238 (2006).

8. Y. Xu, Y. Shi, and S. Ding, "A chemical approach to stem-cell biology and regenerative medicine," Nature 453(7193), 338-344 (2008).

9. H. Fong, K. A. Hohenstein, and P. J. Donovan, "Regulation of selfrenewal and pluripotency by Sox 2 in human embryonic stem cells," Stem Cells 26(8), 1931-1938 (2008).

10. M. F. Pera and P. P. Tam, "Extrinsic regulation of pluripotent stem cells," Nature 465(7299), 713-720 (2010).

11. M. F. Pera, "Defining pluripotency," Nat. Methods 7(11), 885-887 (2010).

12. K. W. Johnson, M. Dooner, and P. J. Quesenberry, "Fluorescence activated cell sorting: a window on the stem cell," Curr. Pharm. Biotechnol. 8(3), 133-139 (2007).

13. R. Bajpai et al., "Efficient propagation of single cells Accutasedissociated human embryonic stem cells," Mol. Reprod. Dev. 75(5), 818-827 (2008).

14. C. Y. Fong et al., "Separation of SSEA-4 and TRA-1-60 labelled undifferentiated human embryonic stem cells from a heterogeneous cell population using magnetic-activated cell sorting (MACS) and fluorescence-activated cell sorting (FACS)," Stem Cell Rev. 5(1), 72-80 (2009).

15. C. R. Nicholas et al., "A method for single-cell sorting and expansion of genetically modified human embryonic stem cells," Stem Cells Dev. 16(1), 109-117 (2007).

16. K. S. Sidhu and B. E. Tuch, "Derivation of three clones from human embryonic stem cell lines by FACS sorting and their characterization," Stem Cells Dev. 15(1), 61-69 (2006).

17. S. H. Yuan et al., "Cell-surface marker signatures for the isolation of neural stem cells, glia and neurons derived from human pluripotent stem cells," PLoS One 6(3), e17540 (2011).

18. L. A. Flanagan et al., "Unique dielectric properties distinguish stem cells and their differentiated progeny," Stem Cells 26(3), 656-665 (2008).

19. J. W. Chan et al., "Label-free separation of human embryonic stem cells and their cardiac derivatives using Raman spectroscopy," Anal. Chem. 81(4), 1324-1331 (2009).

20. F. C. Pascut et al., "Noninvasive detection and imaging of molecular markers in live cardiomyocytes derived from human embryonic stem cells," Biophys. J. 100(1), 251-259 (2011).

21. S. Wachsmann-Hogiu, T. Weeks, and T. Huser, "Chemical analysis in vivo and in vitro by Raman spectroscopy-from single cells to humans," Curr. Opin. Biotechnol. 20(1), 63-73 (2009).

22. W. Denk, J. H. Strickler, and W. W. Webb, "Two-photon laser scanning fluorescence microscopy," Science 248(4951), 73-76 (1990).

23. W. R. Zipfel, R. M. Williams, and W. W. Webb, "Nonlinear magic: multiphoton microscopy in the biosciences," Nat. Biotechnol. 21(11), 1369-1377 (2003).

24. W. R. Zipfel et al., "Live tissue intrinsic emission microscopy using multiphoton-excited native fluorescence and second harmonic generation," Proc. Natl. Acad. Sci. USA 100(12), 7075-7080 (2003). 
25. J. M. Squirrell et al., "Long-term two-photon fluorescence imaging of mammalian embryos without compromising viability," Nat. Biotechnol. 17(8), 763-767 (1999).

26. B. Chance et al., "Intracellular oxidation-reduction states in vivo," Science 137(3529), 499-508 (1962).

27. D. K. Bird et al., "Metabolic mapping of MCF10A human breast cells via multiphoton fluorescence lifetime imaging of the coenzyme NADH," Cancer Res. 65(19), 8766-8773 (2005).

28. D. Chorvat, Jr. and A. Chorvatova, "Multi-wavelength fluorescence lifetime spectroscopy: a new approach to the study of endogenous fluorescence in living cells and tissues," Laser. Phys. Lett. 6(3), 175-193 (2009).

29. H. W. Guo et al., "Reduced nicotinamide adenine dinucleotide fluorescence lifetime separates human mesenchymal stem cells from differentiated progenies," J. Biomed. Opt. 13(5), 050505 (2008).

30. K. König, A. Uchugonova, and E. Gorjup, "Multiphoton fluorescence lifetime imaging of 3D-stem cell spheroids during differentiation," Microsc. Res. Tech. 74(1), 9-17 (2011).

31. A. Uchugonova and K. König, "Two-photon autofluorescence and second-harmonic imaging of adult stem cells," J. Biomed. Opt. 13(5), 054068 (2008).

32. C. Stringari et al., "Phasor approach to fluorescence lifetime microscopy distinguishes different metabolic states of germ cells in a live tissue," Proc. Natl. Acad. Sci. USA 108(33), 13582-13587 (2011).

33. J. Wang et al., "Dependence of mouse embryonic stem cells on threonine catabolism," Science 325(5939), 435-439 (2009).

34. O. Yanes et al., "Metabolic oxidation regulates embryonic stem cell differentiation," Nat. Chem. Biol. 6(6), 411-417 (2010)

35. Y. Guo et al., "Redox regulation of the embryonic stem cell transcription factor oct-4 by thioredoxin," Stem Cells 22(3), 259-264 (2004).

36. R. B. Hamanaka and N. S. Chandel, "Mitochondrial reactive oxygen species regulate cellular signaling and dictate biological outcomes," Trends Biochem. Sci. 35(9), 505-513 (2010).

37. K. Ito et al., "Regulation of oxidative stress by ATM is required for selfrenewal of haematopoietic stem cells," Nature 431(7011), 997-1002 (2004).

38. M. A. Ogasawara and H. Zhang, "Redox regulation and its emerging roles in stem cells and stem-like cancer cells," Antioxid. Redox Signal. 11(5), 1107-1122 (2009).

39. S. Pervaiz, R. Taneja, and S. Ghaffari, "Oxidative stress regulation of stem and progenitor cells," Antioxid. Redox Signal. 11(11), 2777-2789 (2009).

40. J. Smith et al., "Redox state is a central modulator of the balance between self-renewal and differentiation in a dividing glial precursor cell," Proc. Natl. Acad. Sci. USA 97(18), 10032-10037 (2000).

41. R. S. Balaban, S. Nemoto, and T. Finkel, "Mitochondria, oxidants, and aging," Cell 120(4), 483-495 (2005).

42. M. C. Simon and B. Keith, "The role of oxygen availability in embryonic development and stem cell function," Nat. Rev. Mol. Cell Biol. 9(4), 285-296 (2008).

43. C. C. Fjeld, W. T. Birdsong, and R. H. Goodman, "Differential binding of NAD+ and NADH allows the transcriptional corepressor carboxylterminal binding protein to serve as a metabolic sensor," Proc. Natl. Acad. Sci. USA 100(16), 9202-9207 (2003).

44. H. Kamata and H. Hirata, "Redox regulation of cellular signalling," Cell Signal. 11(1), 1-14 (1999).

45. Q. Zhang, D. W. Piston, and R. H. Goodman, "Regulation of corepressor function by nuclear NADH," Science 295(5561), 1895-1897 (2002).

46. M. J. Birket et al., "A reduction in ATP demand and mitochondrial activity with neural differentiation of human embryonic stem cells," J. Cell. Sci. 124(3), 348-358 (2011).
47. M. G. Vander Heiden, L. C. Cantley, and C. B. Thompson, "Understanding the Warburg effect: the metabolic requirements of cell proliferation," Science 324(5930), 1029-1033 (2009).

48. M. A. Digman et al., "The phasor approach to fluorescence lifetime imaging analysis," Biophys. J. 94(2), L14-6 (2008).

49. T. Smits et al., "Correlation between macroscopic fluorescence and protoporphyrin IX content in psoriasis and actinic keratosis following application of aminolevulinic acid," J. Invest. Dermatol. 125(4), 833-839 (2005).

50. N. Billinton and A. W. Knight, "Seeing the wood through the trees: a review of techniques for distinguishing green fluorescent protein from endogenous autofluorescence," Anal. Biochem. 291(2), 175-97 (2001).

51. L. Dong et al., "In vivo and simultaneous multimodal imaging: integrated multiplex coherent anti-Stokes Raman scattering and two-photon microscopy," Appl. Phys. Lett. 97(22), 223702 (2010).

52. K. König and I. Riemann, "High-resolution multiphoton tomography of human skin with subcellular spatial resolution and picosecond time resolution," J. Biomed. Opt. 8(3), 432-439 (2003).

53. D. Schweitzer et al., "Towards metabolic mapping of the human retina," Microsc. Res. Tech. 70(5), 410-409 (2007).

54. J. L. Clokey, "The autofluorescent "lipofuscin granules" in the intestinal cells of Caenorhabditis elegans are secondary lysosomes," Mech. Ageing Dev. 35(1), 79-94 (1986).

55. T. Jung, N. Bader, and T. Grune, "Lipofuscin: formation, distribution, and metabolic consequences," Ann. NY Acad. Sci. 1119, 97-111 (2007).

56. W. L. Rice, D. L. Kaplan, and I. Georgakoudi, "Two-photon microscopy for non-invasive, quantitative monitoring of stem cell differentiation," PLoS One 5(4), e10075 (2010).

57. J. Freubis, S. Parthasarathy, and D. Steinberg, "Evidence for a concerted reaction between lipid hydroperoxides and polypeptides," Proc. Natl. Acad. Sci. USA 89(22), 10588-10592 (1992).

58. M. Riazy et al., "Fluorescent adducts formed by reaction of oxidized unsaturated fatty acids with amines increase macrophage viability," Free Radic. Biol. Med. 51(10), 1926-1936 (2011).

59. R. H. Xu et al., "BMP4 initiates human embryonic stem cell differentiation to trophoblast," Nat. Biotechnol. 20(12), 1261-1264 (2002).

60. J. A. Thomson et al., "Source, embryonic stem cell lines derived from human blastocysts," Science 282(5391), 1145-1147 (1998).

61. Y. M. Cho et al., "Dynamic changes in mitochondrial biogenesis and antioxidant enzymes during the spontaneous differentiation of human embryonic stem cells," Biochem. Biophys. Res. Commun. 348(4), 1472-1478 (2006).

62. C. Allegrucci and L. E. Young, "Differences between human embryonic stem cell lines," Hum. Reprod. Update 13(2), 103-120 (2006).

63. S. M. Schieke et al., "Mitochondrial metabolism modulates differentiation and teratoma formation capacity in mouse embryonic stem cells," J. Biol. Chem. 283(42), 28506-28512 (2008).

64. T. Lonergan, C. Brenner, and B. Bavister, "Differentiation-related changes in mitochondrial properties as indicators of stem cell competence," J. Cell Physiol. 208(1), 149-153 (2006).

65. G. C. Parker, G. Acsadi, and C. A. Brenner, "Mitochondria: determinants of stem cell fate?," Stem Cells Dev. 18(6), 803-806 (2009).

66. C. Xu et al., "Feeder-free growth of undifferentiated human embryonic stem cells," Nat. Biotechnol. 19(10), 971-974 (2001).

67. M. F. Pera et al., "Regulation of human embryonic stem cell differentiation by BMP-2 and its antagonist noggin," J. Cell Sci. 117(Pt. 7), 12691280 (2004). 